\title{
A NEW EFFECTIVE WAY ON VEGETATION MORNITORING USING MULTI-SPECTRAL CANOPY LIDAR
}

\author{
Zhu Bo ${ }^{\mathrm{a}, *}$, Gong Wei ${ }^{\mathrm{a}}$, Shi Shuo ${ }^{\mathrm{a}}$, Song Shalei ${ }^{\mathrm{b}}$ Ma Yingying ${ }^{\mathrm{a}}$ \\ ${ }^{a}$ State Key Laboratory of Information Engineering in Surveying, Mapping and Remote Sensing, Wuhan University, \\ Wuhan, Hubei 430079, People's Republic of China-zhubo125@whu.edu.cn \\ ${ }^{\mathrm{b}}$ Wuhan Institute of Physics and Mathematics, The Chinese Academy of Sciences, P.O. Box 71010, Wuhan 430071, \\ People's Republic of China - songshalei@gmail.com
}

Commission I, I/2

KEY WORDS: Remote Sensing, Vegetation, Monitoring, Global-Environmental, LIDAR, Spectral

\begin{abstract}
:
Airborne Laser Scanning $(A L S)$ has been a well-established tool for the measurement of surface topography as well as for the estimation of biophysical canopy variables, such as tree height and vegetation density. By combining GPS and INS together, ALS could acquire surface information effectively in getting the mass production of DEM and DOM. However, up to now most approaches are built upon single-wavelength Lidar system, which could only provide structure information of the vegetation canopy, the intensity information was rarely used to monitor vegetation growing state as its limitation on spectral characteristics. On the other hand, positive multi/hyper-spectral imaging instruments highly rely on the effects of weather, shadow and the background noise etc. The attempts to fuse single-wavelength Lidar data with multi/hyper-spectral data also been effected this way. Thus, a concept for a multi-wavelength, active canopy Lidar has been tested in this paper. The proposed instrument takes measurement at two vegetationsensitive bands separately at $556 \mathrm{~nm}$ and $780 \mathrm{~nm}$, which, according to the correlation analysis between the wavelengths and biochemical content with plenty of ground $A S D$ reflectance dataset, showed a high correlation coefficient on the chlorophyll concentration as well as nitrogen content. The instrumentation of the multi-wavelength canopy Lidar employs low power, solid and semiconductor laser diodes as its laser source and the receiver consists of two channels, one for 556nm back-scatter signal and the other for $780 \mathrm{~nm}$. The system calibration has also been done by using a standard white board. Multi-wavelength back-scatter signals were collected from a scene consists of stones, healthy broad-leaf trees and unhealthy trees that suffer from disease(part of its leaves were yellow). It is shown that the multi-wavelength canopy Lidar could not only capture the structure information, but also could pick up the spectral characteristics. A further test of three dimensional reconstruction and $S V M$ based classification were also done and the results showed that the spatial resolution could be as high as $5 \mathrm{~mm}$ and the accuracy of classification on those three features (woody/un-woody, healthy/unhealthy) reached to $86 \%$. Therefore, the multi-wavelength canopy Lidar shows its potential capability on vegetation monitoring in a new effective way.
\end{abstract}

\section{INTRODUCTION}

Light Detecting And Ranging(LiDAR) have been widely used in vegetation remote sensing ${ }^{[1,2]}$. Previous LiDAR systems mostly take measurements at one single band which makes it a limitation on intensity using for vegetation monitoring as the lacking of spectral information. On the other hand, results based on positive multi-spectral remote sensors showed a more effective way to do the vegetation monitoring, but it was limited by the solar illumination. Fusions between these two methods make it possible to overcome some of the shortcomings but the effects brought by the weather, shadow etc still exist ${ }^{[3]}$. As a result, the concept for a multi-spectral canopy LiDAR used for vegetation monitoring was proposed recently. Rall and Knox described a nearest dual-wavelength spectral ratio biospheric LiDAR system ${ }^{[4]}$, use which the change of NDVI profile at different season was given out. But it could not detect the range information of the canopy.Tan and Narayanan developed a multi-spectral polarized $\mathrm{LiDAR}^{[5]}$, as the wavelength was not the most sensitive bands of typical vegetation, the capability on vegetation monitoring is limited too. Morsdorf and Nichol et al simulated a four wavelength canopy LiDAR which could monitoring forest canopy growing status ${ }^{[6]}$. However, a real
MWCL instrumentation used for vegetation monitoring as well as structure reconstruction has not been reported so far.

To support the vegetation monitoring mission, a vegetation sensitive two band LiDAR instrument was proposed and tested at Wuhan University. The scope of this study is i): to describe the configuration of this LiDAR system and ii): to complete the preliminary ground experiment setup.

\section{SYSTEM CONFIGURATION}

The Multi-spectral Canopy LiDAR consists of three main parts: (i) the laser source and beam combination sub-system, (ii) the optical receiver light splitting sub-system, and (iv), the data acquisition and processing sub-system. The details of these three main parts will be described in the next sections. The block diagram of the Multi-spectral Canopy LiDAR is shown in Figure 1.

\footnotetext{
* Corresponding author. Current address: State Key Laboratory of Information Engineering in Surveying, Mapping and Remote Sensing, 179 LuoYuRoad Wuhan, Hubei, China

E-mail address: zhubo125@whu.edu.cn
} 


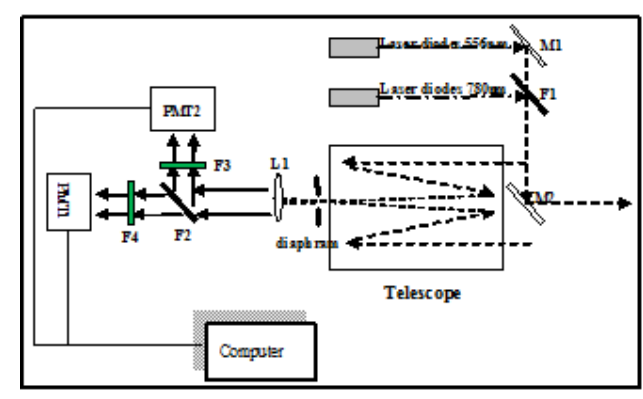

Figure 1 block diagram of the multi-spectral LiDAR system

In Figure 1, the 556nm laser beam was reflected by M1,which has a reflectivity as high as $99.9 \%$. Then combined with $780 \mathrm{~nm}$ laser beam into one single beam after passing through a special filter F1. The back scattered light was collected by a Schmidt-Cassegrain telescope and then send to light splitting system and finally divided into two channels. For each channel, the photonic signals are amplified and converted to electric signals throughout the PMTs, and outputs from the PMTs are A/D converted by a digital oscillograph. Finally, the signals are stored in a computer hard disk, prepared for subsequent data processing.

\subsection{Laser source and beam combination}

The Multi-spectral Canopy LiDAR system employs a low power solid and a semiconductor lasers, both of which are specially designed to meet the requirements for vegetation detection. The key parameters of the two lasers are shown in Table 1.

\begin{tabular}{|l|c|c|}
\hline Parameters & $556 \mathrm{~nm}$ & $780 \mathrm{~nm}$ \\
\hline Power(mW) & 100 & 100 \\
Beam divergence(mrad) & 1.5 & 1.5 \\
Beam diameter(mm) & 2.7 & 2.7 \\
Stability(4h,rms) & $<0.3 \%$ & $<0.3 \%$ \\
Pre-time(min) & 10 & 10 \\
mode & single & single \\
\hline
\end{tabular}

Table 1 key parameters of the multi-spectral canopy LiDAR

Beam combination is the next issue. The advantages to do beam combination are (i) all the lasers must shoot exactly at the same point, otherwise the back-scatter reflectance that the receiver got would come from different object and/or different parts of the object, which means the reflectivity value we measured at the two bands would be mismatched. (ii) the position of the four laser points would change as the detecting distance changes if beam combination was not completed.

Beam combination was done by using a specially designed filterF1 shown in Figure 1.As the output power of both laser diodes were not very strong, the power loss during the optical layout must be considered. Thus, the filter was designed to has a high transmittance at $556 \mathrm{~nm}$ as well as a high reflectance at the band 780nm and was successfully manufactured and applied.

\subsection{Light receiving and spliting}

The backscattered light is collected by a $20 \mathrm{~cm}$ diameter Schmidt-Cassegrain telescope that has an adjustable iris at its focus to limit the receiver field of view. The same specially designed filter used in the beam combination sub-system was employed to complete the light splitting, whose purpose was to split the receiving light that consists of two wavelengths into two independent channels. Before the light was diffused onto each PMT detection plane, a narrow band filter with a FWHM bandwidth of $10 \mathrm{~nm}$ was fixed ahead, which would greatly reduce the background noise. The signals were amplified and photoelectric converted by the PMTs and A/D converted by a digital oscillograph.

\subsection{Data acquisition and processing}

A LABVIEW program was carried out to automatically complete the data acquisition and processing. We use a $2 \mathrm{G} \mathrm{Hz}$ digital oscillograph to digitalize the signal collected by the receiving sub-system and the digitalized data was send and stored on the computer hardware disk. During the data processing, a simple wavelet method was used to do the denoise and all de-noised data was calibrated and transformed into reflectance data by making use of a standard white board. The reflectance calculated according to equation 1 :

$$
R_{\text {laser }}=\frac{A_{o b}}{A_{w b}} \times 100 \%
$$

where $R_{\text {laser }}$ refers to the reflectance of measured objects, $A_{o b}$ refers to the amplitude measured of the objects and $A_{w b}$ refers to the amplitude measured of the standard white board.

\section{EXPERIMENT SETUP AND DISCUSSION}

The Multi-spectral LiDAR system was tested on the ground by measuring a scene that contains healthy and plastic leaves. The goal of this experiment is to test the capability of the multispectral Canopy LiDAR system on vegetation discrimination. The measured features was shown in Figure 2:

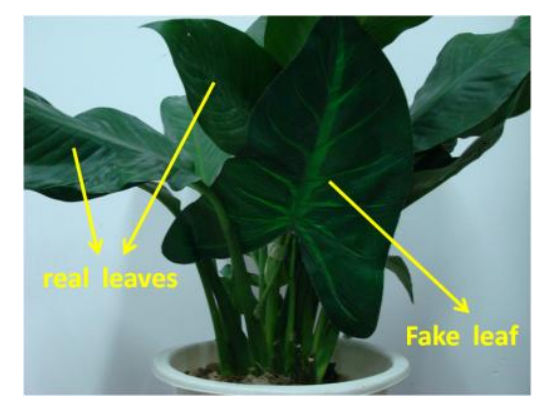

Figure 2 measured features, which contains one single fake leaf and the others are healthy leaves.

A push broom scanning was done through the multi-spectral Canopy LiDAR instrument. Both the rang information and two band spectral information were derived at the same time. A 3 dimensional reconstruction based on the LiDAR cloud points 
was done with a TIN model and a simple 4 class classification was also accomplished through a SVM algorithm based on the spectral information. The results were shown in Fig4(a),(b) separately:

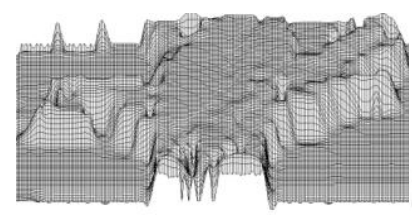

(a)

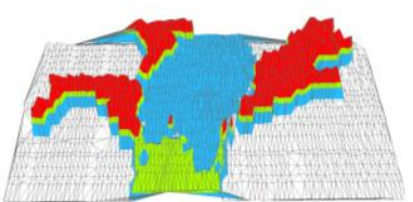

(b)
Figure 4 Test results based on multi-spectral canopy lidar:(a) the $3 \mathrm{D}$ reconstruction based on the measured cloud points and

(b) an SVM classification results based on the spectral information

In . 4(a), the structure profile of the vegetation was successfully acquired through a TIN model. The discrimination on the complicated scene reached to about $3 \mathrm{~mm}$. In Figure4(b), a further analysis on the spectral space showed a high precision on feature classification. Most of the healthy green leaves was correctly identified as show in red region the green region refers to the stems of the plant, the white region refers to the background signals and more importantly, the blue region, which refers to the fake leaf, was also highly discriminated by the two band spectral information. That means the Multispectral Canopy LiDAR works quite well on vegetation discrimination as we can still distinguish between the fake leaf and the real leaves even if the color and shape of the fake leaf are almost the same as the healthy green leaves.

\section{CONCLUSION}

In this paper, a prototype of a Multi-Wavelength Canopy LiDAR system was designed and implemented to identify its capability on vegetation canopy profiling and feature classification. The results show that the Multi-spectral Canopy LiDAR system could not only capture the structure information of the objects (vegetation included), but also could get the intensity spectral information. Such a instrument of the Mulltispectral Canopy LiDAR system was potentially shown its capabilities on vegetation monitoring needs. However, as there are only two bands employed in the Multi-specral Canopy LiDAR system, the spectral information is still not enough to do further quantitative crop management analysis, we could add more necessary bands to enrich the spectral information. Meanwhile, a more reasonable and effective way in calibration system is also needed in consideration.

\subsection{Acknowledgements}

This work was supported by 973 Program (2009CB723905), the NSFC (41101334, 10978003, 41127901).

\section{References:}

[1] Wehr and Lohr, Airborne laser scanning-An introduction and overview, ISPRS Journal of Photogrammetry and Remote Sensing. Vol. 54 (1999), p. 68-82

[2] K. Kraus and N. Pfeifer, Determination of terrain models in wooded areas with airborne laser scanner data, ISPRS
Journal of Photogrammetry and Remote Sensing, vol. 53 (1998), p. 193-203

[3] Koetz, B., Sun, G., Morsdorf, F., Ranson, K., Kneubuhler, M., Itten, K., et al. Fusion of imaging spectrometer and lidar data over combined radiative transfer models for forest canopy characterization. Remote Sensing of Environment, Vol.106 (4), p.449-459.( 2007)

[4] Rall, J., \& Knox, R. Spectral ratio biospheric lidar. 22nd International Laser Radar. Conference (ILRC 2004), Proceedings of the Conference held 12-16 July 2004 in Matera, Italy ESASP-561. (pp. 831).

[5] Tan, S., \& Narayanan, R. M. Design and performance of a multi-wavelength airborne polarimetric lidar for vegetation remote sensing. Applied Optics, Vol. 43(11), 2360-2368.( 2004)

[6] Morsdorf, F., Nichol, C. Assessing forest structural and physiological information content of multi-spectral LiDAR waveforms by radiative transfer modeling, Remote Sensing of Environment ,Vol. 113(2009), 2152-2163 